\title{
Augenmerk auf Ernährung und Infektionen
}

Mit einem besonderen Augenmerk auf die erhöhten Infektionsrisiken von Patienten mit Colitis ulcerosa (CU) und den Aspekt Ernährung haben Experten unter Federführung der Deutschen Gesellschaft für Gastroenterologie, Verdauungs- und Stoffwechselkrankheiten (DGVS) die Leitlinie für die Behandlung der CU aktualisiert.

In Österreich sind Schätzungen zufolge etwa 40.000 Personen betroffen, in Deutschland geht man von etwa 150.000 Erkrankten aus, die unter den Symptomen krampfartige Bauchschmerzen, Blut im Stuhl und immer wieder Durchfällen in ihrer Lebensqualität oft schwer beeinträchtigt sind. Meist im jungen Erwachsenenalter, nicht selten auch schon bei Jugendlichen und Kindern, setzt der Beginn der Erkrankung ein, die in Schüben verläuft und die Betroffenen in der Regel ein Leben lang begleitet. Obwohl die Krankheit nicht selten ist, dauert es bei vielen CU-Patienten aber noch immer lange, bis sie die richtige Diagnose und eine adäquate Therapie erhalten. Die neue Leitlinie der DGVS zur Diagnostik und Behandlung der CU rückt nun beispielsweise die Rolle der Ernährung in ein neues Licht, denn abgesehen vom Stillen gibt es keine wissenschaftlich belegte Ernährungsform, die das Risiko für die Entstehung einer CU-Erkrankung reduziert.

\section{Umwelt und Hygiene als Auslöser}

Ein wichtiger Auslöser der Erkrankung und eine Erklärung für die Zunahme der CU seit der Mitte des 20. Jahrhunderts scheint in veränderten Umwelt- und Hygienebedingungen der modernen Zivilisation zu liegen. CU tritt eher in Industrieländern als Entwicklungsländern, eher bei Städtern als in der Landbevölkerung auf. „Die Zunahme der Fallzahlen in den vergangenen Jahrzehnten führte zudem zur Theorie, dass der Auslöser für die Erkrankung auch in den modernen Ernährungsgewohnheiten zu finden sein könnte", sagt Prof. Dr. Axel Dignaß, Med. Klinik I, Agaplesion Markus Krankenhaus Frankfurt, einer der Koordinatoren der Leitlinie. Studien hätten diesen Zusammenhang bisher allerdings nicht bestätigt. Einzige Ausnahme sei das Stillen: Kinder, die mindestens sechs Monate lang gestillt wurden, haben ein um fast ein Viertel reduziertes Risiko, später an CU zu erkranken, als nicht oder nur kurz gestillte Kinder.

Bei bereits bestehender CU kommt der Ernährung hingegen eine große Bedeutung zu. „Wegen der wiederkehrenden Durchfälle und der Schädigung der Darmschleimhaut haben die Patienten ein hohes Risiko für eine Mangelernährung", stellt Prof. Dr. Torsten Kucharzik, Klinik f. Allg. Innere Medizin und Gastroenterologie, Klinikum Lüneburg, fest, der die Aktualisierung der Leitlinie ebenfalls als Koordinator betreut hat. Oft schwächen die Komplikationen, die durch den Nährstoffmangel hervorgerufen wurden, die Patienten mehr, als die Darmentzündung selbst. Besonders Kinder weisen häufig - in bis zu $85 \%$ - Zeichen einer Mangelernährung auf. Neben starken Proteinverlusten wirken sich auch eine zu geringe Versorgung mit Mikronährstoffen wie Eisen, Vitamin D, Folsäure oder Zink negativ auf Wachstum und Entwicklung aus. Die Versorgung mit Nährstoffen sollte daher regelmäßig überprüft und fehlende Nährstoffe als Tablette oder Infusion zugeführt werden, empfiehlt die Leitlinie.

\section{Immunsystem außer Kontrolle}

Die Ursachen für die Entstehung einer CU sind nach wie vor nicht vollständig geklärt, zentral scheint jedoch eine Fehlsteuerung des Immunsystems zu sein. Die Patienten werden daher meist mit immunsuppressiven Medikamenten behandelt. Damit steigt jedoch die Gefahr von bakteriellen oder viralen Infektionen deutlich an.,,Besonders die Kombination mehrerer Medikamente stellt ein Problem dar", so Kucharzik. In Studien hatten Patienten, die mehrere solcher Medikamente einnehmen mussten, ein 14,5-fach erhöhtes Infektionsrisiko. Die Mediziner raten daher dazu, noch vor Beginn der Therapie den Impfstatus der Patienten zu überprüfen und fehlende Impfungen nachzuholen. Auch die jährliche Grippeimpfung sei für immunsupprimierte Patienten dringend zu empfehlen.

Wien klin Mag 2019 · 22:111 https://doi.org/10.1007/s00740-0190272-3

(c) Springer-Verlag GmbH Austria, ein Teil von Springer Nature 2019

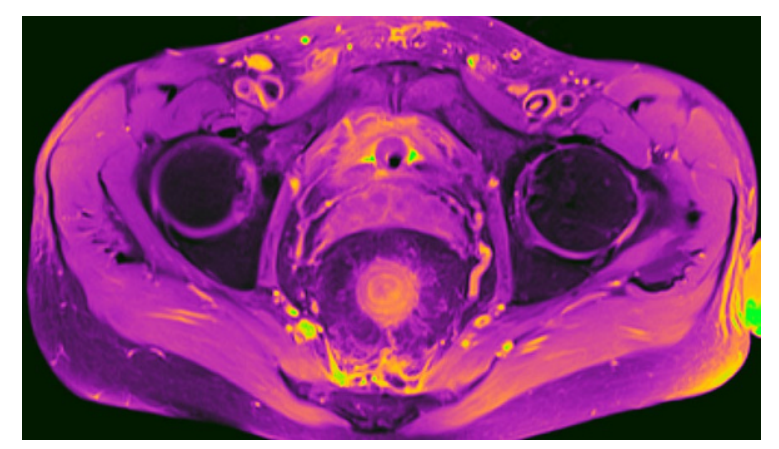

○ [M] A. o. Univ.-Prof. Dr. Helmut Schöllnast, Universitätsklinik für Radiologie, Medizinische Universität 